\title{
Unveiling Su Aurigae in the near Infrared: New high spatial resolution results using Adaptive Optics
}

\author{
Abhijit Chakraborty, \& Jian Ge \\ Department of Astronomy \& Astrophysics, 525 Davey Laboratory, Pennsylvania State University, \\ University Park, PA 16802 \\ abhijit@astro.psu.edu \\ jian@astro.psu.edu
}

\begin{abstract}
We present here new results on circumstellar nebulosity around SU Aurigae, a T-Tauri star of about 2 solar mass and 5 Myrs old at $152 \mathrm{pc}$ in the J, H and $\mathrm{K}$ bands using high resolution adaptive optics imaging (0!30) with the Penn state IR Imaging Spectrograph (PIRIS) at the 100 inch Mt. Wilson telescope.

A comparison with HST STIS optical (0.2 to 1.1 micron) images shows that the orientation of the circumstellar nebulosity in the near-IR extends from PAs 210 to 270 degrees in $\mathrm{H}$ and $\mathrm{K}$ bands and up to 300 degrees in the $\mathrm{J}$ band. We call the circumstellar nebulosity seen between 210 to 270 degrees as 'IR nebulosity'. We find that the IR nebulosity (which extends up to 3.5 arcsecs in $\mathrm{J}$ band and 2.5 arcsecs in the $\mathrm{K}$ band) is due to scattered light from the central star. The IR nebulosity is either a cavity formed by the stellar outflows or part of the circumstellar disk. We present a schematic 3-dimensional geometrical model of the disk and jet of SU Aur based on STIS and our near-IR observations. According to this model the IR nebulosity is a part of the circumstellar disk seen at high inclination angles. The extension of the IR nebulosity is consistent with estimates of the disk diameter of 50 to $400 \mathrm{AU}$ in radius, from earlier $\mathrm{mm}, \mathrm{K}$ band interferometric observations and SED fittings.
\end{abstract}

Subject headings: stars: individual (SU Aurigae) - stars: pre-main-sequence - stars: formation - scattering -techniques: high angular resolution - techniques: image processing

\section{Introduction}

SU Aurigae (SU Aur) is a T-Tauri star located in the Taurus-Aurigae complex of dark molecular clouds at a distance of $152 \mathrm{pc}$ (de Warf et al. 2003, Hipparcos catalog). Its spectral type is G2III, mass $\sim 2 M_{\odot}$ and age is about 4 to 5 million years (de Warf et al. 1998, de Warf et al. 2003). Recent observations by Nadalin et al. (2000) have shown short time variability in the B band magnitude that they attribute to proto-planetary materials orbiting in the circumstellar disk. Petrov et al. (1996) have found existence of a gas outflow from the star from their spectroscopic results.

The HST/STIS coronagraphic observations by Grady et al. (2002) from 0.2 to 1.0 microns have revealed fan like structures extending up to a distance of 12-15 arcsecs in the west to south-west direction. These, according to the authors, are mainly reflection nebulae scattering the light of the central star. In addition they also detected streaming filamentary structures going radially outwards from the star which could be due to gas outflow either from the star or from the parent molecular cloud.

Our motivation comes from the STIS images of SU Aur, and from the fact that no report of high spatial resolution near-infrared images of the star could be found in the literature to this date. Therefore, we observed the source in the nearinfrared (J, $\mathrm{H}$ and $\mathrm{K}$ band images) using adaptive optics and applied the technique of PSF subtrac- 
tion to investigate the circumstellar region of $\mathrm{SU}$ Aur with high spatial resolution $\left(0^{\prime \prime} \cdot 25\right.$ to $\left.00^{\prime \prime} 30\right)$. In this paper we present the results of our investigation (of SU Aur). Section 2 describes observations and data analysis procedure, section 3 introduces the results, in section 4 we discuss our results and present our conclusions in section 5 .

\section{Observations and Data Analysis}

\subsection{Observations}

SU Aurigae was observed in the $\mathrm{J}, \mathrm{H}$ and $\mathrm{K}$ photometric bands during October 2002 at the 100 inch telescope of Mt.Wilson using its natural star Adaptive Optics (AO) system (Shelton et al. 1995) and the Penn state near-IR Imager and Spectrograph (PIRIS, Ge et al. 2003). The detector is a $256 \times 256$ PICNIC array with 40 micron pixels. The plate scale is $00^{\prime \prime} 082$ per pixel providing a field of view of $21^{\prime \prime} 0$. PIRIS is also equipped with cold pupil masks in the pupil plane to reduce the thermal background (particularly in the $\mathrm{K}$ band).

The night of observations (19 Oct. 2002) was photometric with sub-arcsec seeing and the FWHM of star's Point Spread Function (PSF) after the AO correction was measured to be 0 "' 25 . Seven images of SU Aur with 60 second exposures in each photometric band were recorded when the object was near zenith (air mass close to 1.00). Two PSF stars were also observed namely BD +40 248 (Spectral type = G2V, PSF1) and Gl 46.1 (Spectral type $=$ G5V, PSF2). Gl 46.1 was observed 3.5 hours before the SU Aur observations with similar air mass (or close to the zenith) while the other PSF star BD +40 248 was observed immediately before the SU Aur observations but at a higher air mass (1.6). This allowed us to determine the stability of the PSF during the night of observations as the telescope tracks different sources over different air masses. We find that the FWHMs of the PSF stars were 0.'25 and 0". 29 respectively, and the overall shape did not change by more than $5 \%$ between the two PSF stars. In fact another star Gl 230 (of spectral type G2) was observed (at air mass $=1.1$ ) as a part of a different program immediately after the SU Aur observations by the same instrument and its PSF has a FWHM of $0^{\prime \prime} 28$ and has similar shape.

The PSF stars were selected based on their similar brightness and spectral type to SU Auri- gae. For instance, the $\mathrm{J}, \mathrm{H}, \mathrm{K}$ magnitudes of $\mathrm{BD}$ +40 248 are 5.76, 5.35, 5.27 and Gl 46.1 are 5.50, $4.96,4.75$ respectively (from 2MASS All Sky Point Source Catalog). From the same catalog the SU Aur J, H, K magnitudes are 7.20, 6.56 and 5.99 respectively. Three exposures of 1, 10, 20 and 30 seconds in each photometric bands $(\mathrm{J}, \mathrm{H}$ and $\mathrm{K})$ of the PSF stars were taken. This procedure facilitates the scaling of the PSF stars with respect to the PSF of SU Aur. Six sky images were also taken before and after the observations of all the sources. These sky frames were combined to obtain sky frames for sky subtraction. Normalized twilight sky images were used as flat frames in the $\mathrm{J}, \mathrm{H}, \mathrm{K}$ bands.

\subsection{Data Analysis \& the Procedure of Scaled PSF Subtraction}

Data analysis was performed using various IRAF tasks. All images went through a standard pipeline of sky subtraction and flat field correction before the specialized tasks of PSF matching and subtraction. Figure 1 shows J band image of SU Aur before the psf subtraction and a scaled and azimuthally smoothed (in sectors of 15 degrees) mean image of PSF star. Figure 2 shows a radial plot (azimuthally averaged over 360 degrees) of the mean PSF star in Figure 1.

Since SU Aur is known to possess a large extended nebulosity (Grady et al. 2002, Woodgate et al. 2003, Nakajima \& Golimowski 1995), the PSF matching of SU Aur with that of the PSF stars is not a straight forward task. The usual procedure of matching the wings of the PSF of the object (SU Aur) with that of the PSF stars was not possible because of the presence of extended emission from SU Aur. Therefore, an alternate procedure was adopted: 1) To get rid of the high frequency noise and to generate a symmetric smooth PSF function, each PSF image was azimuthally averaged over sectors of 15 degrees. 2) A comparison of the relative brightness of SU Aur with that of the PSF stars using their apparent magnitudes in the respective bands was made. 3) The intensity of the PSF star images were scaled to SU Aur. For instance, BD +40 248 (PSF1) is 1.2 mag or 3 times brighter than SU Aur in the $\mathrm{H}$ band, so the intensity from a 20 second exposure frame of PSF1 star should match that of 60 second exposure of SU Aur. 
The third step was repeated for all exposures times of the PSF stars by multiplying with suitable factors. Thus, the brightness of both the PSF stars (PSF1 \& PSF2) were scaled to that of SU Aur and were cross checked by subtracting one scaled PSF star from the other until minimal intensity residuals were found in the scaled PSF1-PSF2 images. The residual patterns of PSF1-PSF2 subtraction are shown in Figure 3. Due to the difference in spectral type of stars (in the present case PSF1 is G2 and PSF2 is G5) the difference in slope of the continuum across the filter bandwidth leaves a diffraction pattern as residuals. For example the work of Krist et al. (1998) on PSF subtraction of one normal star from the other using the HST data has clearly demonstrated these effects.

At the end of the third step we obtained two scaled PSF star images which are similar. The final PSF star image was constructed by taking mean of the two scaled PSF star images. We note here that the $\mathrm{x}-\mathrm{y}$ positions of the PSF image were matched with that of SU Aur up to a one-fifth $(1 / 5)$ of a pixel for good subtraction. Figure 4 shows PSF subtracted images of SU Aur using the same contrast level as that of Figure 3.

The HST-STIS data (0.2 to 1.1 micron, Grady et al. 2002) in Figure 4 was downloaded from the HST archive, analyzed by the standard STSDASIRAF pipeline routines and then over-plotted with the contour levels of the $\mathrm{J}$ band intensity levels from $15.0 \mathrm{mag}$ to $9.0 \mathrm{mag}$ at an interval of $0.5 \mathrm{mag}$. We note that we did not perform PSF subtraction on the STIS image since the STIS image is only used for comparison of brighter regions between the optical and near-infrared nebulosity.

Figure 5 shows mean surface brightness of the disk with respect to the distance from the star in the J,H and $\mathrm{K}$ bands. The mean surface brightness was calculated by summing up the disk brightness in the azimuthal direction between PAs 210 to 310 degrees and dividing it by the total number of spatial resolution element (in this case $0.3 \times 0.3$ arcsecs, $4 \times 4$ pixel elements). It is clear that the procedure of PSF subtracting images taken with the Mt.Wilson AO system can be used to detect faint extended emission up to contrast levels of $10^{-4}$ per resolution element (which is $0^{\prime \prime} 3 \times 0^{\prime \prime} .3$ ) beyond 1 arcsec from the central bright star. The crosses with error bars show the intensity of the extended nebulosity and the lines with error bars show the residuals of PSF1 - PSF2. The accuracy of the photometry is measured to be $0.2 \mathrm{mag}$. The lines in the J,H,K bands therefore show instrument detection limits. In the present work we will only consider the extended structures of SU Aur which are beyond 1 arcsec.

\section{Results}

As evident from Figures 4 and 5 the brightness varies between 12 to $15 \mathrm{mag} /$ resolution element. The brightness of the circumstellar nebulosity scales with the distance from the star as $1 / r^{1.2}$ to $1 / r^{2}$ between 1 to 4 arcsec (where $\mathrm{r}$ is the distance from the star in arcsecs) and maximum extent up to $3^{\prime \prime} .5$ in the J band and less than $2 . .5$ in the $\mathrm{K}$ band. The size of the circumstellar nebulosity remains intermediate in the $\mathrm{H}$ band. In the $\mathrm{J}$ band, nebulosity is prominent from PAs 210 to 300 degrees, and faintly visible at PAs from 50 to 115 degrees.

The geometry of the extended nebulosity is more intriguing when compared with the STIS image (Figure 4). Although the overlapping contours of the J band image intensity show regions bright in the STIS image, it also shows a region which is between PAs 210 to 270 degrees. In $\mathrm{H}$ and $\mathrm{K}$ bands only the latter region is seen clearly and will be referred to as 'IR nebulosity' hence forth.

Grady et al. (2002) attributed the radially streaming filamentary structure (seen in the STIS image) at $\mathrm{PA}=295$ to 300 degrees as the blue jet emerging from the star. It is worth mentioning here that ground based coronagraphic images in $\mathrm{R}$ and $\mathrm{I}$ bands using adaptive optics also show similar features (Nakajima \& Golimowski, 1995). These coronagraph images show both the radially outward streaming nebulosity seen in the STIS image as well as the region between PAs 200 to 250 degrees in our near-IR images. Their work shows brightness of about 17 and $16.5 \mathrm{mag} / \operatorname{arcsec}^{2}$ in $\mathrm{R}$ and I bands respectively at a distance of 3-4 arcsec from the star between $\mathrm{PA}=200$ to 250 degrees and about $14.5 \mathrm{mag} / \operatorname{arcsec}^{2}$ (in R and I) between $\mathrm{PA}=260$ to 300 degrees.

\section{Discussion}

SU Aur is known to possess a large accretion disk of size up to $400 \mathrm{AU}$ and an outer envelope (Akeson et al. 2002, hereafter ACBC, Oliveira et 
al. 2000). Both ground based and HST/STIS coronagraphic images of SU Aur (Nakajima \& Golimowski 1995, Grady et al. 2002) in the optical wavelengths have shown spatially resolved outflows and a reflection nebula mainly due to scattered star light. In the near-IR, however, the PSF subtracted J,H,K images of SU Aur (present work) reveal an extended nebulosity at angles $(\mathrm{PA}=210$ to 270) where there is little optical emission (Figure 4, also Nakajima \& Golimowski 1995). The region where the strongest optical nebulosity is seen is between PAs $=295$ to 310 (Grady et al. 2002). Therefore, the regions probed in the nearIR are different from those in the optical wavelengths. We discuss below about the IR nebulosity.

There can be two explanations for the IR nebulosity: a) the region of IR nebulosity is the opening of a cavity in the parent molecular cloud seen almost edge on and formed by the stellar outflow (Stapelfeldt et al.1998); b) it is the part of the circumstellar disk itself seen at high inclination angles ( $\geq 65$ degrees). In either case the region may suffer greater extinction in the optical wavelengths (see Cotera et al. 2001). We present the likelihoods of both the scenarios below with emphasis on the second one (that the IR nebulosity is the part of the disk seen in the scattered light of the central star). We also present a geometrical model based on the disk assumption.

The IR nebulosity can be a cavity formed by the bipolar outflows from the star (Stapelfeldt et al. 1998). Scattered light model calculations on TTau and IRAS $04016+2610$ by Wood et al.(2001) have shown that cavities formed by multiple stellar outflows and misaligned circumstellar disks can be seen in the scattered light of the central star up to a radii of 500AU. The observed IR nebulosity in SU Aur could be a cavity formed by the blue jet. However, a very large opening angle of greater than 70 degrees for the blue jet (Grady et al. 2002) would be necessary to explain the PA of the IR nebulosity.

The IR nebulosity could be the part of the circumstellar disk seen almost edge-on. The geometrical model describing the orientation of the disk with respect to the observer is described by ACBC. ACBC calculated the most probable physical parameters of the disk like the position angle $\mathrm{PA}$, angle of inclination and extent of the disk from
$\mathrm{K}$ band and $\mathrm{mm}$ interferometry and SED curve fittings. They found that the disk outer radius could be 50 to $400 \mathrm{AU}\left(0^{\prime \prime} 3\right.$ to $\left.2^{\prime \prime} 6\right)$ ) with an angle of inclination of about 62 degrees and a position angle of 130 degrees. ACBC state that their data set on SU Aur is not sufficient to constrain the model parameters and the quoted disk parameters by ACBC are the most probable ones from the model fittings. However, according to STIS coronagraphic observations (Grady et al. 2002) the blue jet is at PA $=295$ degrees and a probable red jet is at $\mathrm{PA}=114$ degrees.

We propose a schematic 3-dimensional geometrical model for SU Aur based on our near-IR observations and the STIS observations (Grady et al. 2002). The model (not to scale) is shown in Figure 6. The disk is seen close to edge-on (angle of inclination $=65$ degrees or greater, from Grady et al. 2002 and ACBC) and at an azimuthal angle of about 180 degrees (see Figure 6). Thus one side of the disk surface faces towards the observer along with one of the jets (the blue jet). The part of the disk surface facing towards the observer could be seen as the IR nebulosity and the remaining part of the disk could be obscured due to high optical extinction (Cotera et al. 2001). According to these authors scatterd star light through the disk can suffer from very high extinction of $A v=80$ or more (also see Wood et al. 2001).

Further, if the IR nebulosity is part of the optically thick disk seen almost edge on then we can compare the total flux of the IR nebulosity with that of the model of ACBC. Such a comparison will also indicate if the IR nebulosity is due to scattered light from the star. Figure 7 of ACBC shows the flux (in Jansky) of the theoretical model of the disk that was used to fit the SED. We estimate an upper limit for the total flux (in Jansky) of the IR nebulosity in each band, assuming an area covered by an annular ring of radii 1 and 2.6 arcsecs and compared these values with the model of ACBC. These are listed below as (values from present work) $/($ model values of ACBC) in J, $\mathrm{H}, \mathrm{K}$ respectively: $(0.8) /(0.10),(0.2) /(0.5)$, and $(0.11) /(1.1)$.

If the model values are reliable then it is possible that in the $\mathrm{J}$ band the observed flux of the IR nebulosity is dominated by the star scattered light from the disk and the stellar jet (as in the optical wavelengths of the STIS image) rather than 
the disk flux itself. The comparison between the $\mathrm{H}$ band fluxes are the closest, which may be a coincidence. It is most likely that the total flux in the $\mathrm{H}$ band is dominated by the dust scattering. In the $\mathrm{K}$ band we find that the observed disk flux and the model values differ by an order of an magnitude. The scattering in the $\mathrm{K}$ band is less compared to the $\mathrm{J}$ band and optical wavelengths and is expected to be dominated by the disk's thermal emission. ACBC predicts the disk temperature to be 55 to $150 \mathrm{~K}$ at $10 \mathrm{AU}$ and is expected to fall at larger radii. Since the present work is sensitive only to distances greater than $1 \operatorname{arcsec}(160 \mathrm{AU})$, we are unable to see the thermal component from the disk and measure only the scattering component.

\section{Conclusions}

We have presented here PSF subtracted high spatial resolution images ( 0. ". 30 arcsecs) of the circumstellar region of SU Aurigae (between 1 to 4 arcsecs) in the J,H and $\mathrm{K}$ bands. These images show a distinct region bright in the near-IR which we call the IR nebulosity. The IR nebulosity is prominent between position angles 210 degrees to 270 degrees and extends up to 3.5 arcsecs in the $\mathrm{J}$ band and 2.5 arcsecs in the $\mathrm{K}$ band. We present two scenarios about the nature of the IR nebulosity: a) that it could be a cavity formed by the stellar outflows or b) that it is a part of the circumstellar disk observed at high inclination angles ( $\geq 65$ degrees). We favor the later case more and we present a schematic 3-dimensional geometrical model describing the orientation of the disk and jet of SU Aur with respect to the observer. However, more observations are necessary with a large telescope equipped with AO and perhaps with coronagraphs and polarimetry to resolve spatial structures close to the star (down to 0.1 arcsecs) for detailed modeling.

We thank the Mt. Wilson staff for their help during the AO observations, and Mr. Junfeng Wang for assisting the observations. We also acknowledge Dr. Lee Hartmann and Dr. Nuria Calvet for stimulating discussions. We thank the anonymous referee for his/her comments which significantly improved the quality of the paper. We also thank Julian van Eyken and John Debes for proofreading the manuscript carefully. This work is supported by National Science Foundation with grant AST-0138235, and NASA grants NAG5-12115 and NAG5-11427. This publication makes use of data products from the Two Micron All Sky Survey, which is a joint project of the University of Massachusetts and the Infrared Processing and Analysis Center/California Institute of Technology, funded by the National Aeronautics and Space Administration and the National Science Foundation. HST-STIS data presented in this paper were obtained from the Multimission Archive at the Space Telescope Science Institute (MAST). STScI is operated by the Association of Universities for Research in Astronomy, Inc., under NASA contract NAS5-26555. Support for MAST for non-HST data is provided by the NASA Office of Space Science via grant NAG5-7584 and by other grants and contracts.

\section{REFERENCES}

Akeson, R.L. et al. 2002, ApJ, 566, 1124 (ACBC)

Cotera, A.S. et al. 2001, ApJ, 556, 958

de Warf, L.E. et al. 1998, IAU Information Bulletin on variable stars, No. 4551

de Warf, L.E. et al. 2003, ApJ, 590, 357

Ge, J., Chakraborty, A., Debes, J.H., Ren, D., \& Friedman, J. 2003, Proc. SPIE 4841, 1503

Grady, C. et al. 2002, AAS, 199, 60.15

Krist, J.E., et al. 1998, PASP, 110, 1046

Nakajima, T., \& Golimowski, D.A. 1995, AJ, 109, 1181

Nandalin, I. et al. 2000, AU Information Bulletin on variable stars, No. 4987

Oliveira, J.M., et al. 2000, in Disks, Planetesimals and Planets, ASP Conference Series, ed: F. Garzn, C. Eiroa, D. Winter, and T.J. Mahoney, also astroph/0004377

Petrov, P.P. et al. 1996, A\&A, 314, 821

Shelton et al. 1995, proc. SPIE, 2534, 72

Stapelfeldt, K.R. et al. 1998, ApJ, 502, L65

Wood, K. et al. 2001, ApJ, 561, 299 
Woodgate, B. et al. 2003, proc. SPIE, 4860, 10

This 2-column preprint was prepared with the AAS LATEX macros v5.2. 


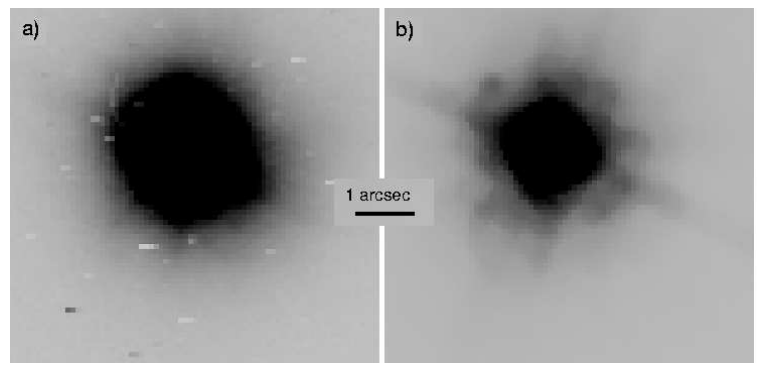

Fig. 1.- a) J band image of SU Aur before the PSF subtraction. b) A scaled and azimuthally smoothed (in sectors of 15 degrees) mean PSF star image in the $\mathrm{J}$ band. North is top and East is to the left.

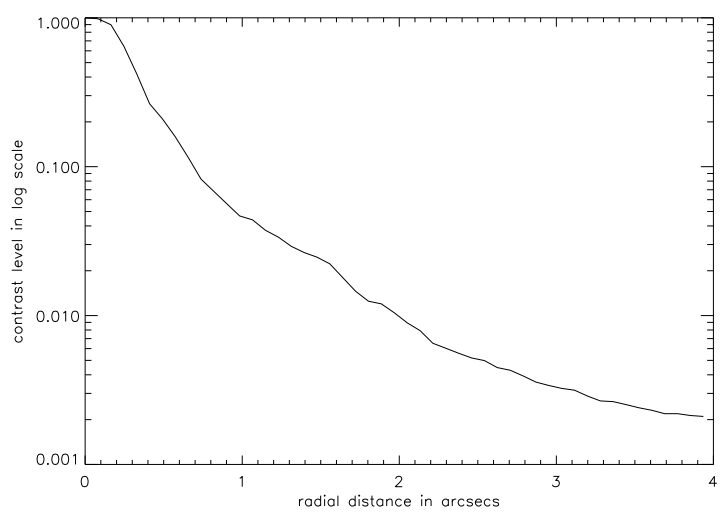

Fig. 2.- A radial plot (azimuthally averaged over 360 degrees) of the mean PSF star in Figure 1 showing the contrast levels in log scale versus radial distance in arcsecs.

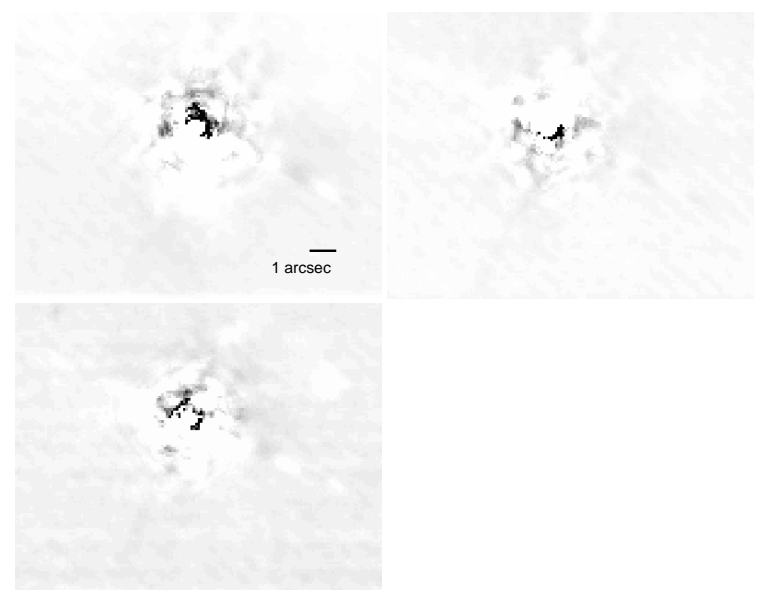

Fig. 3.- PSF1 (BD+40 248) subtracted from PSF2 (Gl46.1) J(top left), H(top right), and K(bottom left) band images. See text for details. North is up and east is to the left. The grey scale levels are same as those in the $\mathrm{J}, \mathrm{H}$ and $\mathrm{K}$ images of SU Aur in Figure 4. 


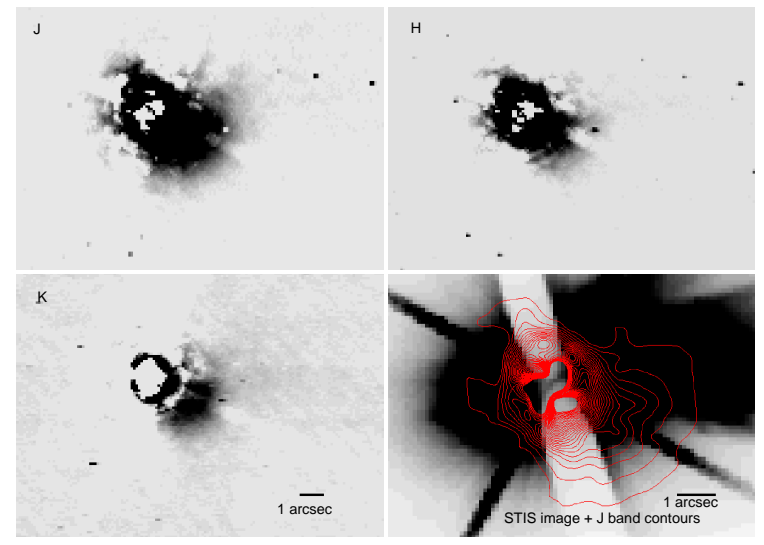

Fig. 4.- PSF subtracted J,H,K band images of SU Aur showing the associated circumstellar nebulosity. See text for details. The fourth image (bottom right) is the STIS coronagraphic image (0.2-1.1microns) from the HST archives (also Grady et al. 2002), and over plotted are the contours from the J band image with contour levels from 15.0 to $9.0 \mathrm{mag} / \mathrm{resolution}$ element (see figure 5) with an interval of 0.5 mag. North is up and east is to the left. The grey scale levels are same in the $\mathrm{J}, \mathrm{H}, \mathrm{K}$, and STIS images. Also note that the position of the diffraction spikes is along the ridges at about $\mathrm{PA}=250$ degrees seen across the nebulosity.

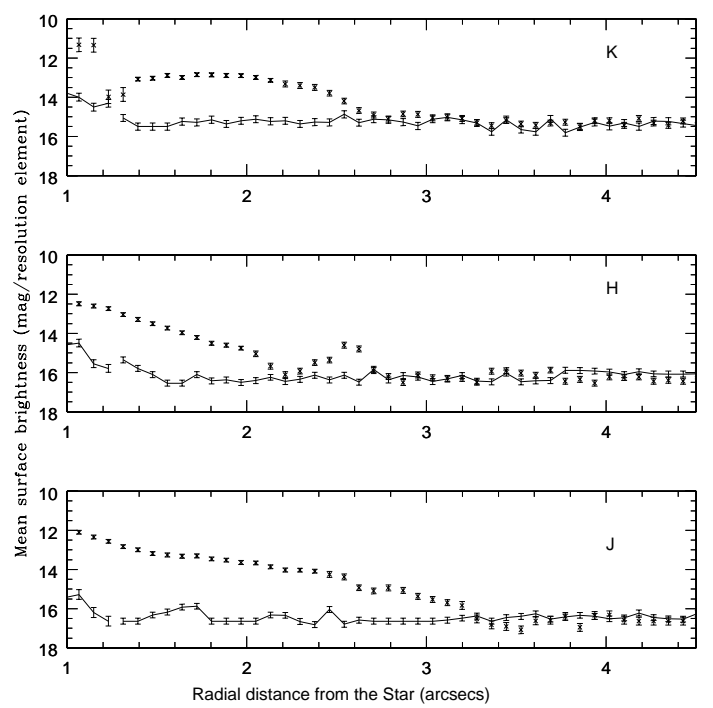

Fig. 5.- Mean surface brightness (mag/resolution element, averaged azimutally between PAs 210 to 310 degrees) of the disk with respect to the projected distance (arcsec) from the central star in J,H and K bands. Here one resolution element is $0.3 \times 0.3 \mathrm{arcsec}^{2}$. The crosses with error bars show the mean brightness of the disk while the simple lines with error bars show the intensity level from the PSF1 - PSF2 subtracted images. 


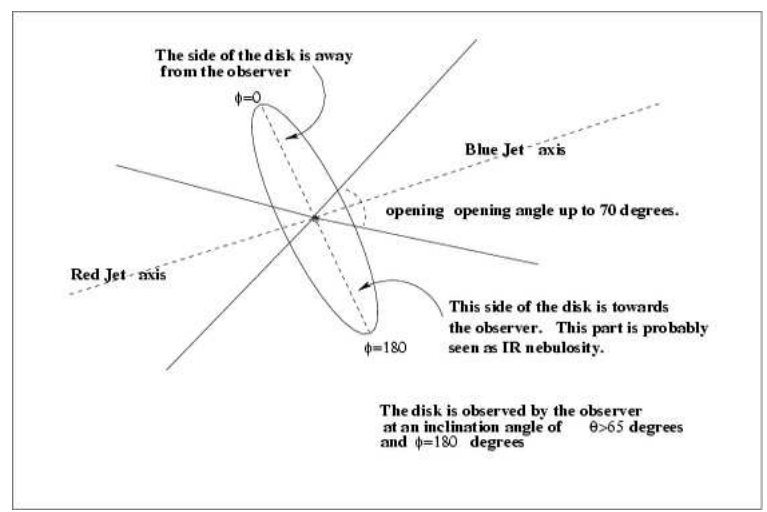

Fig. 6.- Schematic 3-dimensional geometrical model (not to scale) of SU Aur based on STIS and present NIR observations. The observer is along the direction of $\phi=180$ degrees with the observer being able to view the face of part of the inclined disk. This region of the disk may be seen as the IR nebulosity. 